\title{
Integration of reaction and separation with lipases: An overview ${ }^{1}$
}

\author{
Ana L. Paiva, F. Xavier Malcata \\ Escola Superior de Biotecnologia, Universidade Católica Portuguesa, 4200 Porto, Portugal
}

\begin{abstract}
Integration of reaction and separation steps in processes involving lipases is currently undergoing fast progress because it provides a convenient way to alleviate limiting kinetic factors that are common in biological reactions, viz. decreased product inhibition, increased product solubility, shifted chemical equilibria, increased selectivity, and reduced number of downstream unit operations and process streams (with concomitant increases in the efficiency of separation). It is the aim of this work to briefly review the processes that have been developed to achieve integration of lipase-catalytic action with separation. These processes fall within one of the following categories: solid/liquid systems (e.g. membrane reactors for microencapsulated lipases in reversed micelles), solid/fluid systems (e.g. supercritical reaction/extraction processes), solid/liquid/vapour systems (e.g. reactive distillation), and solid/vapour systems (e.g. evaporative esterification).
\end{abstract}

Keywords: Enzyme; Catalysis; Separation; Integration

\section{Introduction}

The use of biotechnological routes to achieve results that have traditionally been obtained only via bulk chemical processes has been expanding steadily. One of the most important examples is the use of enzyme-catalyzed reactions, which occur to high rates at room temperature and pressure, conditions that minimise thermal degradation of labile compounds and avoid use of chemicals with a potential for pollution. Furthermore, enzyme-mediated processes allow one to obtain qualitatively more pure compounds than chemical syntheses because the latter are often unspecific and generate a variety of by-

\footnotetext{
* Corresponding author.

' Part of the information conveyed in this paper was presented in the International Workshop on Microbial Lipases in the Biocatalysis. Rome (Italy), April 11-13, 1996.
}

products. However, two major drawbacks are associated with enzymatic processes: conversion is usually not high, and so dilute product solutions are obtained; and intrinsically low productivities are often achieved because crude (commercial) enzyme preparations are employed rather than pure (analytical) enzymes. These drawbacks arise from low concentrations of active enzyme, kinetic and thermodynamic inhibition of the enzyme by reactant(s) and/or product(s), and degradation of the enzyme either by heat (thermal deactivation) or by compounds present in the reaction mixture (chemical deactivation). In order to avoid the problem of low concentrations of enzyme in the reaction medium, immobilization has become a common practice; genetic manipulation has also allowed design of enzymes with cnhanced activity and stability which can be overproduced by microorganisms at relatively high purity. However, 
the problem of reactant and/or product inhibition is more difficult to solve, even though improvement of existing, and introduction of novel, separation techniques have been attempted consistently in the near past.

Traditionally, separation processes have been carried out whenever a reaction, due either to kinetic or thermodynamic constraints, does not approach the extension desired within a given time frame, or when products obtained do not have the degree of purity required by the preset specifications. Separation at the post-reactional level often requires considerable amounts of energy and a set of cascaded steps. In order to improve effectiveness of separation and hence reduce costs, the possibility of continuously removing the product(s) formed during reaction has been under scrutiny. This approach allows achievement of higher yields by preventing thermodynamic equilibria to be attained, and higher rates by not allowing product(s) that contribute to enzyme inhibition/deactivation to be present at high concentrations. Such in situ separation of products from unreacted reactants, although positive from the point of view of commercial feasibility of enzyme-catalyzed processes, adds considerably to the technical complexity of the process and so makes modelling and prediction more difficult.

Lipases are hydrolases which (apparently) have been tailored by nature to hydrolyse unsoluble triglycerides with concomitant production of free fatty acids and glycerol. However, such enzymes also catalyze the reverse reaction (esterification) and, in general, display catalytic activity towards ester bonds involving a large variety of alcohols and carboxylic acids. Integration of separation with reaction catalyzed by lipases is relevant because accumulation of water as reaction product (thermodynamically) prevents extensive esterification and also promotes deactivation of the lipase, whereas accumulation of free fatty acids as reaction products (kinetically) inhibits hydrolysis. Integration of reaction and separation in non-conventional biocatalytic systems (i.e. microaqueous mixtures) with li- pases as catalysts is thus particularly interesting due to the catalytic behaviour of these enzymes, and has consequently been undergoing fast progress; advantages claimed for such integration include decrease of product inhibition, increase of product and substrate solubilities via use of multiphasic systems, shift of chemical conversion in the esterification direction, increase of selectivity towards a desired product taking advantage of the conformational role of organic solvents upon lipases, and dramatic reduction of the number of downstream separation operations and process streams.

It is the aim of this paper to critically review and discuss processes encompassing integration of separation with reaction catalyzed by lipases, a general family of nonconventional innovative processes termed extractive biocatalysis.

\section{Integrated reaction/separation processes with lipases}

Native (and recombinant) lipases catalyze hydrolysis and its reverse, esterification, as well as sequential combinations thereof generally termed interesterifications (acidolysis, alcoholysis, and transesterification). Integrated processes which have to date used lipases fall within one of the following categories: solid/liquid systems (e.g. membrane reactors for microencapsulated lipases in reversed micelles), solid/fluid systems (e.g. supercritical reaction/extraction processes), solid/liquid/vapour systems (e.g. biocatalytic distillation), and solid/vapour systems (e.g. evaporative esterification). These four types of systems are depicted in Fig. 1, whereas integrated processes are compared with classical unit operation processes in Fig. 2.

\subsection{Solid/liquid systems}

Use of membrane reactors constitutes an attempt to integrate catalytic conversion, product separation, and product enrichment steps into one single apparatus, thus resulting in greater productivity and lower costs [1]. 
There is a large variety of synthetic membranes commercially available with suitable specifications for use in membrane bioreactors. Semi-permeable membranes may be used to retain the enzyme (by plain mechanical confinement due to size exclusion, or actual immobilization due to adsorption or covalent binding), and thus separate it from the reactants/products at the reactor outlet. Membranes may also be designed for selective removal of one product relative to others or to reactant [2]. Important characteristics to be taken into account when choosing a membrane include morphology, porosity, pore size distribution, molecular weight cut-off, chemical resistance, biological inertness, tolerance to temperature, $\mathrm{pH}$ and pressure, and price [3]. Several membrane modules can be found in the market: flat-sheet (straight laid out and spiral wound), tubular, and hollow fibers [4]. The type of membrane reactors using lipases and their applications have been reviewed elsewhere [5], as well as the strategies for modelling of such reactors $[1,6]$.

Most reactions catalyzed by lipases are carried out in biphasic media due to the low solubilities of reactants and/or products in conventional aqueous media. An alternative approach is encapsulation of lipases in reversed micellar media (e.g. Refs. [1,7-20]) followed by suspension in organic solvents where those reactions can be carried out in a "pseudo" uniphasic system.

Encapsulation consists on confinement of the

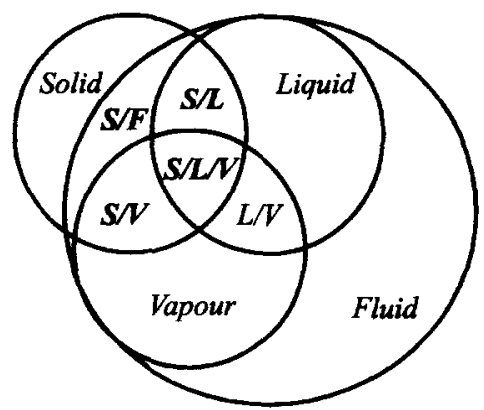

Fig. 1. Schematic representation of the multiphasic nature of integrated reaction/separation processes with lipases.

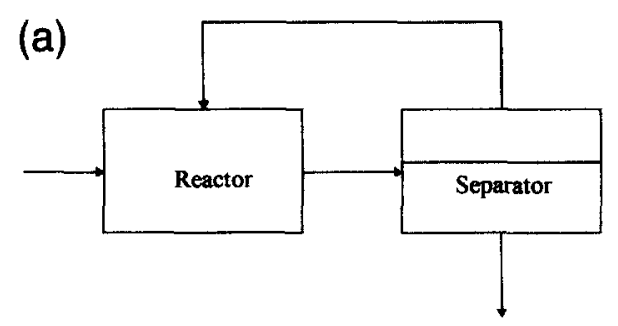

(b)

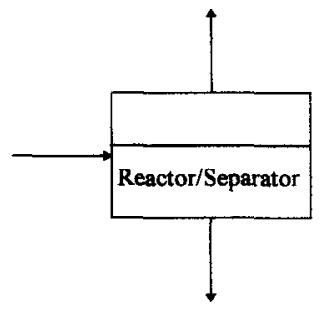

Fig. 2. Schematic representation of (a) a classical unit operation process; (b) an integrated reaction/separation process.

lipase solution within small capsules enclosed by a polymer or a surfactant, whose diameters can range from 0.01 up to $300 \mu \mathrm{m}$ [21-23]. Such capsules provide an aqueous environment for the lipase thus reducing the problem of interfacial denaturation that prevails in twophase systems. They also possess the advantages of a potentially high interfacial specific area (which is kinetically stable and hence does not require mechanical input for maintenance), and a facilitated enzyme recovery (since the lipase is contained in capsules). Permanent and non-permanent polymeric membranes can offer wide ranges of surface area and reactant specificity because, in some cases, the membrane can be manufactured so as to admit only some substrates and, thus, selectively exclude others. The encapsulation membrane creates an extra resistance to mass-transfer, and so the 'effectiveness factor' for encapsulated lipases may be quite small unless capsules with a very small diameter can be produced (as in the case of reversed micelles). On the other hand, encapsulation is not applicable when the size of the substrate molecule approaches that of the lipase [22].

Reversed micelles are aqueous dispersions stabilized in an organic solvent by a surfactant 
which can cither be cationic, anionic or nonionic, and usually has a polar head and a nonpolar tail; in this type of liquid immobilization, the lipase remains in the aqueous core of the reversed micelle while the substrates and products partition between the aqueous and the organic phases. The amphiphilic nature of the surfactant molecule causes its self association in a reverse-micellar solution with the polar head of the surfactant directed towards the aqueous core of the micelle and the hydrophobic tail extending into the continuous organic phase. The essentially spherical semipermeable membranes of reversed micelles have pores which allow small substrates and product molecules to enter and leave the capsule, respectively, but prevent lipases and large molecules from permeating $[21,22,24]$. The size and structure of reverse micelles make them attractive for integrated processes $[25,26]$, and several reviews on reversed micellar systems using enzymes that are concerned with structural and catalytic properties are available [27-38]. Major disadvantages of using reversed micelles are that: (i) only low molecular weight substrates can be considered; (ii) occasionally inactivation of enzyme occurs during the immobilization procedure; (iii) a high enzyme concentration is required for micelle formation; (iv) the enzyme may actually become incorporated into the membrane wall; and (v) it is difficult to recover the product(s) from the surfactant-containing organic solvent. Therefore, and despite its intrinsic potential for continuous operation, catalysis in reversed micellar systems has been mainly performed in batch type reactors; exceptions encompass immobilization of a lipase in liposomes which, in turn, were solubilized in reversed micelles and used for the continuous glycerolysis of olive oil in an ultrafiltration cell [39].

Development of reactor designs which enable continuous reaction and product scparation is hence one of the current critical issues in reversed micelle technology $[1,40]$. Several attempts have been made to use membrane reactors to carry out enzymatic reactions with re- versed micelles [39-47]; however, the membranes used were not capable of completely retaining the surfactant monomers and the hydrated micelles, therefore allowing contamination of the product streams [40-42], an observation that is not surprising if one considers the specific dynamic characteristics of reversed micellar systems. Even if size exclusion of whole reversed micelles seems reasonable, retention of the rather small surfactant monomers usually found in dynamic equilibrium with micelles by an ultrafiltration membrane is highly improbable. Additionally, it is necessary to consider the stability of reversed micelles to shear forces usually found in membrane apparata; deformation of the micellar structure, or even eventual destruction, will decrease their retention by ultrafiltration [40]. Nevertheless, ultrafiltration membrane reactors seem to be the most appropriate reactor configuration for the confinement of encapsulated lipases in reversed micellar media and simultaneous (partial) product separation $[5,39,40,45,48-52]$.

One of the most effective membrane bioreactor for simultaneous reaction catalyzed by a lipase and separation of products in a reversed micellar medium has been proposed by Prazeres et al. $[12,40,51,52]$ (see Fig. 3). The main goal was to confine the encapsulated lipase in the retentate side of the membrane where reaction occurs, and to concomitantly recover products on the permeate side; Chromobacterium visco-

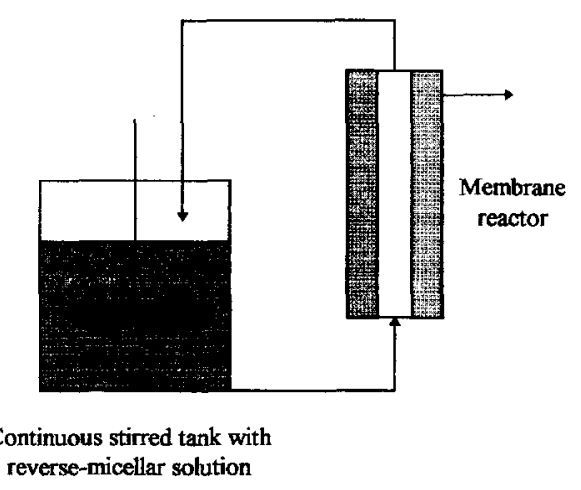

Fig. 3. Continuous reaction and separation in reversed micelle/membrane systems. 
sum lipase encapsulated in AOT (dioctyl sodium sulfosuccinate)/isooctane reversed micelles was thus successfully used for hydrolysis of triolein/olive oil mixtures. The substrate/product pair (water/glyccrol) is located inside the micelles while the other substrate/product pair (triolein/oleic acid) is dispersed in the organic solvent and merged as cosurfactant in the AOT shells of the micelles $[9,10]$. An ultrafiltration tubular membrane module (operated either in batch or continuous mode) was then used to retain the lipase-filled micelles and separate the reaction products. The organic solvent compatibility of the membrane used, its successful regeneration with fresh lipase after each operation, complete retention of lipase, and almost complete separation of oleic acid were the major advantages of this membrane system. Since partial adsorption of lipase onto the membrane was observed, one concluded that a great part of the reaction actually occurred in the vicinity of the membrane $[1,40]$ rather than in the bulk retentate side. Continuous operation of such type of reactor proved to be feasible as long as water-filled micelles were supplemented to the reactor together with the substrate/solvent solution $[12,40,41,52]$. Permeation by distorted reversed micelles and substrate were noticed as drawbacks for successful operation of the reactor; in fact, presence of AOT in the permeate stream makes it more complex to further separate products [53] but is essential for separation of glycerol and for control of its content under continuous operation conditions [40].

\subsection{Solid/fluid systems}

Supercritical fluids, SCF or simply fluids, form a special class of non-conventional solvents. A fluid is called supercritical when both its temperature and its pressure are above their critical values, a state where such interfacial properties as surface tension vanish. In this state, fluids have transport properties that are intermediate between those of a gas and those of a liquid; e.g. fluids possess liquid-like solvent strength but gas-like mobility, permeation ability, diffusivity, and viscosity. When the reduced temperature of a fluid is slightly above unity and its reduced pressure ranges from 1 to 6 , the density of the fluid changes sharply from low gas-like values (at low pressures) to high liquid-like values (at higher pressures). Such high density values explain the solvent strength of a SCF, while the easiness of retrograding between low and high densities via changes in pressure explains the usefulness of SCF Extraction (SCFE) as a separation process.

Since SCFE with common fluids is usually performed at low temperatures (a very significant parameter for thermosensitive molecules such as lipases and reactants/products thereof), the associated consumption of thermal energy is low; solvents can be easily recovered from the mixture for reuse due to their high volatility; high selectivity towards different solutes can be modelled via changes in processing conditions; and most SCF used as solvents are non-toxic. There are, however, several disadvantages associated with SCFE, viz. the high operating pressures required (which demand powerful compressors and thus high fixed costs), the flammability of some SCF (e.g. propane), the need for batch operation mode (which leads to a multiplicity of extractors in order to achieve high productivity), and the still essentially empirical selection of solvents and cosolvents.

A large number of applications in the food, pharmaceutical, and polymer industries exist for SCFE [54] since thermosensitive and highly pure products (with a high market value) are ubiquitously involved. However, use of SCF is not restricted to separation processes; such reaction processes as production of polymers and biomolecules in SCF allow not only running the reaction in a single homogeneous phase (thus eliminating interfacial mass-transfer limitations) but also controlling the reaction extent via adjustment of temperature or pressure (thus changing the equilibrium constant and/or the equilibrium conversion). The use of lipases acting as catalysts in non-aqueous media (such as those 
provided by SCF) has becn described in the literature since they exhibit certain advantages, viz. low rates of thermal decay, extensive solubilization of hydrophobic compounds, and effective shifting of conversion towards synthesis rather than hydrolysis [54].

Lipase reactions in supercritical carbon dioxide $\left(\mathrm{SCCO}_{2}\right)$, the most commonly employed SCF, have been first studied by Nakamura et al. in 1985 [55] for the case of interesterification of triglycerides, and have more recently received the attention of several other researchers [5564]. However, there is still a major lack of fundamental information pertaining to biocatalysis in supercritical fluids to permit one to rationally (and a priori) select a SCF for a desired enzymatic reaction [65].

Nowadays a trend exists towards use of supercritical fluids to bring about reaction coupled with separation. Experiments performed under supercritical conditions in well-stirred reactors [66] and in fixed bed reactors [67] provide mass transfer rates that are much greater than in standard liquid-solid or gas-solid systems, probably because of the favourable contribution arising from natural convection in supercritical fluids. A unique advantage of integrating reaction and separation in SCF pertains to variable selectivity towards recovery; after reaction, critically located sequential depressurization stages allow products and non-reacted substrates to be obtained independently and without any trace of solvent. Combes [68] has developed a continuous reaction/separation process carried out in $\mathrm{SCCO}_{2}$ for the synthesis of ethyl oleate from oleic acid and ethanol catalyzed by a lipase from Mucor miehei immobilized by ion exchange onto a macroporous resin (see Fig. 4). The loss of activity after $6 \mathrm{~d}$ (ca. $20 \%$ at $60^{\circ} \mathrm{C}$ ) was approximately the same in $\mathrm{SCCO}_{2}$ and $n$-hexane, and the effect of temperature in $\mathrm{SCCO}_{2}$ was consistent with the classical thermal denaturation scheme. No effect of pressure in the range $13-18 \mathrm{MPa}$ on deactivation could be detected, which thus created expectancy of operating a continuous reactor for long periods

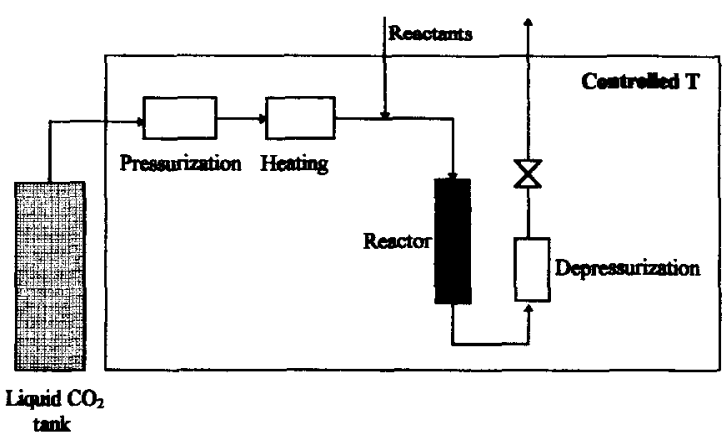

Fig. 4. Continuous reaction and separation in supercritical carbon dioxide.

of time. A continuous reaction/separation process with solvent recycling using a tubular packed reactor has been successfully operated over a period of $15 \mathrm{~h}$ at $40^{\circ} \mathrm{C}$ and $15 \mathrm{MPa}$, with about $95 \%$ oleic acid esterified with ethanol in ca. $1 \mathrm{~h}[69,70]$. Performance of the same model reaction in $\mathrm{SCCO}_{2}$ and $n$-hexane indicated that the solvents are similar from the point of view of enzyme activity, but $\mathrm{SCCO}_{2}$ is better in terms of post-reactional separation.

\subsection{Solid/liquid / vapour systems}

Another process that integrates reaction and separation is reactive distillation. This concept was first applied in 1923 [71,72] for the continuous production of ethyl acetate using sulfuric acid as homogeneous catalyst. Two main reasons accounted for development of such process for performance of esterification: (i) low molecular weight esters are, in general, much more volatile than the alcohol and carboxylic acid involved in their synthesis, and (ii) separation of esters from the reaction medium is often difficult by simple distillation because of formation of azeotropes. Employment of reactive distillation for reactions using a solid catalyst which can be confined to the distillation column by physical methods for reuse and, hence, will not appear in the product stream, is a more recent development [73].

The impetus for integration of reaction with separation conveyed by distillation when per- 
forming lipase-mediated transformations (using the enzyme as a solid catalyst) in esterification reactions has derived from realization that accumulation of water as reaction product, apart from contributing to deactivation of the enzyme, favours the undesired reverse reaction (hydrolysis). Since in synthesis of low molecular weight esters water is, in general, less volatile than both the alcohol and the ester involved as reactants, its fractional removal from the reaction medium may be achieved by preferential vaporization at room pressure and performance of the chemical reaction onto the condensed liquid obtained therefrom rather than onto the full reaction medium. The operation of a distillation column as a chemical reactor offers distinctive advantages over the usual process of a reactor followed by a distillation column since, in this integrated unit, two processes are occurring simultaneously in a single piece of equipment with concomitant reduction of capital and operation costs (due to partial elimination of pumping, piping, and instrumental devices) and increase in overall conversion due to in situ removal of reaction products while reaction is still in progress [74]. Paiva and Malcata [74,75] have reported use of a batch still to which a distillation column was connected; on the top of the distillation column, a total condenser was fitted, and the liquid reflux coming therefrom (richer in the more volatile compounds than the mixture in the still) then passed to a packed bed reactor where a lipase from Mucor miehei, immobilized by ion-exchange onto a macroporous resin was located; the outlet liquid stream from the reactor was then recirculated to the top of the distillation column (see Fig. 5).

This particular configuration overcomes the problem of the high temperatures usually involved in distillation processes carried out at room pressure (and consequent high rates of lipase deactivation), as well as the problem of catalyst dilution (via enzyme immobilization). By using this innovative reaction/separation system, the authors concluded that conversion constraints were reduced to a great extent; con-

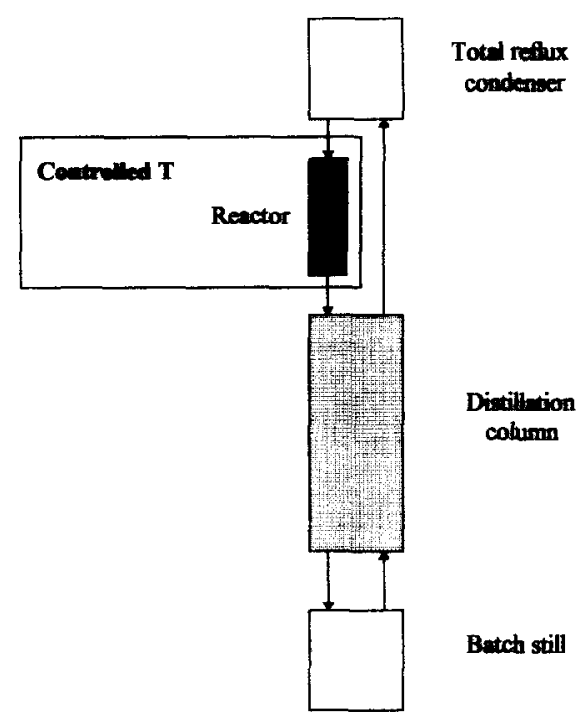

Fig. 5. Continuous reaction and separation in biocatalytic distillation.

versions were higher when amounts of acid in the initial mixture were larger; and lower temperatures led to higher conversions to esters (the magnitude of this effect increased as the molar fraction of acid in the initial mixture increased). The higher productivities observed with this solid/liquid/vapour system result probably from alleviation of equilibrium constraints (e.g. preferential removal of water tends to shift the reaction in the hydrolysis direction) and kinetic constraints (e.g. preferential removal of acetic acid tends to decrease inhibition of enzyme action).

\subsection{Solid/vapour systems}

Despite the fact that organic solvents solubilize essentially apolar compounds and often decrease their viscosity, they may be considered as a contaminant or even a pollutant in the final product stream, in addition to acting as denaturants for enzymes in the case they are not strictly hydrophobic. Therefore, solvent-free alternatives have been sought. One such alternative encompasses use of a gas-solid system for single-step biotransformations [76-90]. Parvaresh et al. [84] reported use of this new technique with a lipase as a lyophilized powder 


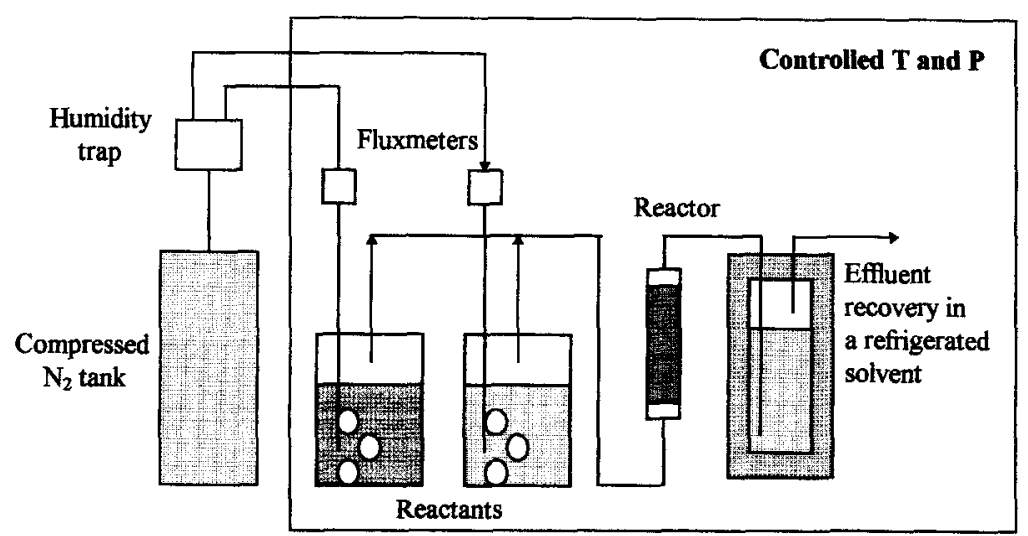

Fig. 6. Continuous reaction and separation in evaporative esterification.

for the performance of transesterification reactions on substrates in vapour form (see Fig. 6). Such type of integrated reactor/separator presents several advantages, viz.: (i) operation in a continuous mode is possible and, therefore, substrates can enter and products leave the reactor in a continuous fashion; (ii) there is no addition of solvents and, therefore, no need for solvent recycling; (iii) pure products are easily collected by split recondensation, and hence it is possible to reuse untransformed substrates; (iv) previous immobilization of the biocatalyst is not necessary since it is used in a precipitated form; ( $v$ ) the inlet stream to the reactor is enriched in the more volatile substrates; (vi) enzymes in a dehydrated form are more resistant to thermoinactivation, thus allowing use of higher temperatures with concomitant substantial increase in reaction rates; (vii) the absence of water makes it possible to perform unconventional reactions catalyzed by hydrolases (due to their modified selectivity); and (viii) operation at relatively high temperatures prevents microbial contamination and hence the need for sterilization.

Experiments with porcine pancreatic lipase and Fusarium solani cutinase (a lipase analogue) in catalysis of transesterification reactions between various gaseous substrates in such continuous packed bed gas-solid reactor permitted one to conclude that enzyme activity depends on the nature of the esters and on the chain lengths of the alcohols (i.e., the reaction rate is higher when the chain length is shorter, but the enzyme stability is higher when the chain length is longer) [84]. In order to access the effect of humidity on performance of lipases (recall that these enzymes require interfacial activation), experiments using glass beads coated with lipases and suspended in mixtures of substrate and water vapours over a range of humidity from 56 to $100 \%$ suggested the following order of reaction rates at decreasing water activity levels: hydrolysis > alcoholysis > transesterification $>$ esterification [90]. Such studies have shown that lipases are active on gaseous substrates even at hydration levels below the monolayer coverage of the protein. However, there is an antagonistic effect concerning water activity: the higher the water activity, the higher the enzyme-catalyzed reaction rate, but also the higher the enzyme deactivation rate. The major problem for this configuration is that most substrates suitable for such process exhibit rather high boiling points (250$300^{\circ} \mathrm{C}$ ) at normal pressure, which are incompatible with the heat lability of those compounds and of lipase itself.

\section{Thermodynamic and kinetic assessments of integrating reaction and separation}

The issue of extractive reaction, or reaction coupled with separation, has been claimed to 
provide kinetic and thermodynamic enhancements in the case of biochemical processes (e.g. Refs. [91,92]). However, the terminology used to express possible increases in product yields is often misleading.

Paiva and Malcata [93] tackled the topic of thermodynamic enhancement of integration via consideration of a theoretical cascade of reactors and separators taken to the limit of an infinite number of units coupled with assumption of the simple model system of a binary ideal reactant/product mixture at constant pressure and temperature. Under these postulates, they proved that no thermodynamic improvement of the overall process is brought about by integration of reaction and separation when compared with the classical unit operation approach. Such conclusion, which pertains solely to compositional changes, was reached based on either calculation of the price in Gibbs' free energy currency that must be paid by the surroundings to the system in order to recover an extra mole of pure product or calculation of the income of the surroundings in (overall) Gibbs' free energy currency per unit amount of (separation) Gibbs' free energy invested by the surroundings in the system. This conclusion can be extrapolated for multicomponent ideal solutions and binary (and multicomponent) non-ideal solutions.

Using a similar theoretical rationale, Paiva et al. [94] investigated the effect of kinetic improvement on the chemical processing of an ideal binary mixture when enzyme-catalyzed reaction is integrated with physical separation. The objective function was the reduction in total processing time (i.e. the sum of the time required by chemical reaction to occur in the whole set of reactors with the time required by the partial separation to occur in the whole set of separators) brought about by integrating reaction and separation steps with respect to a traditional system for a predefined final conversion; the model selected was a reversible Uni-Uni reaction following the classical MichaelisMcnten mechanism, and the separation process was assumed to be controlled by mass transport and provide a constant degree of purification. The kinetic enhancement associated with integration of reaction and separation depends on the values of the equilibrium constant $\left(K_{\mathrm{eq}}\right.$, a measure of the degree of thermodynamic inhibition), the final conversion of substrate desired $\left(\chi_{\mathrm{f}}\right)$, the Michaelis-Menten constant for the product $\left(K_{\mathrm{mp}}\right.$, a measure of the degree of kinetic inhibition), the extent of separation in a single step $(\xi)$, and the ratio of time scales for molecular transport and chemical reaction $(\Xi)$. It was claimed that integration is not advisable under strong kinetic and/or thermodynamic inhibition since it leads to higher processing times than the unit operation approach does. In general, integration provides decrease in overall processing time when $K_{\text {mp }}$ decreases and/or $\Xi$ decreases and/or $\xi$ decreases and/or $K_{c q}$ increases and $/$ or $\chi_{\mathrm{f}}$ increases. Parameter $\xi$ did not seem to play a major influence in the general shape of the curve of total time versus the remaining parameters, except when $K_{\text {eq }}$ and $K_{\text {mp }}$ are large. It was interesting to note that cascading (or partially integrating) the reaction/separation system with similar values of $\xi$ brings about higher degrees of product recovery from the binary mixture. On the other hand, intermediate conversions coupled with large $\Xi$, large $K_{\text {eq }}$, small $K_{\mathrm{mp}}$ and small $\xi$ give rise to the existence of an optimum (finite) degree of cascading, thus providing evidence that full integration may not be the best option in terms of overall processing time

\section{Acknowledgements}

Partial funding for this research was provided through grant PRAXIS/2/2.1/BIO/34/94 (Portugal, Project Process Integration: Extractive Biocatalysis). Funding for author A.L.P. was provided through a Ph.D. Fellowship by JNICT (Portugal, CIENCIA BD/2081/92 - IF and PRAXIS XXI BD/5568/95). 


\section{References}

[1] D.M.F. Prazeres, F. Lemos and J.M.S. Cabral, in: A.R. Moreira and K.K. Wallace (Eds.), Computer and Information Science Applications in Bioprocess Engineering (Kluwer Academic Publishers, Dordrecht, 1996) p. 191.

[2] A. van der Padt and K. van 't Riet, in: C.A. Costa and J.M.S. Cabral (Eds.), Chromatographic and Membrane Processes in Biotechnology (Kluwer Academic Publishers, Dordrecht, 1991) p. 443.

[3] J.R. Hildebrandt, in: C.A. Costa and J.M.S. Cabral (Eds.), Chromatographic and Membrane Processes in Biotechnology (Kluwer Academic Publishers, Dordrecht, 1991) p. 363.

[4] J.A.L. Santos, M. Mateus and J.M.S. Cabral, in: C.A. Costa and J.M.S. Cabral (Eds.), Chromatographic and Membrane Processes in Biotechnology (Kluwer Academic Publishers, Dordrecht, 1991) p. 177.

[5] D.M.F. Prazeres and J.M.S. Cabral, Enzyme Microb. Technol. 16 (1994) 738.

[6] F.X. Malcata, Trends Chem. Eng. 1 (1993) 359.

[7] D. Han, D.Y. Kwon and J.S. Rhee, Agric. Biol. Chem. 51 (1987) 615.

[8] D. Han, J.S. Rhee and S.B. Lee, Biotechnol, Bioeng. 30 (1987) 381.

[9] D.G. Hayes and E. Gulari, Biotechnol. Bioeng. 35 (1990) 793.

[10] D.G. Hayes and E. Gulari, Biotechnol. Bioeng. 38 (1991) 507.

[11] E.A. Malakhova, B.I. Kurganov, A.V. Levashov, I.V. Berezin and K. Martinek, Appl. Biochem. Biotechnol. 12 (1983) 182.

[12] D.M.F. Prazeres, F. Lemos, F.A.P. Garcia and J.M.S. Cabral, J. Chem. Technol. Biotechnol. 53 (1992) 159.

[13] S.W. Tsai and C.L. Chiang (1991), Biotechnol. Bioeng. 38 (1991) 206.

[14] P.D.I. Fletcher, B.H. Robinson, R.B. Freedman and C. Oldfield, J. Chem. Soc. Faraday Trans. I 81 (1985) 2667.

[15] P.S. Chang and J.S. Rhee, Biocatalysis 3 (1990) 343.

[16] P.K. Schmidli and P.L. Luisi, Biocatalysis 3 (1990) 367.

[17] R. Scartazzini and P.L. Luisi, Biocatalysis 3 (1990) 377.

[18] D. Han, P. Walde and P.L. Luisi, Biocatalysis 4 (1990) 153.

[19] A. Hochkoeppler and S. Palmieri, Biocatalysis 3 (1990) 357.

[20] J.P. Chen and H. Pai, J. Food Sci. 56 (1991) 234.

[21] J.M.S. Cabral, in: B. Mattiasson and O. Holst (Eds.), Extractive Bioconversions (Marcel Dekker, New York, 1991) p. 207.

[22] J.F. Kennedy and J.M.S. Cabral, in: W.H. Scouten (Ed.), Solid Phase Biochemistry: Analytical Synthetic Aspects (Wiley, New York, 1983) ch. 7, p. 253.

[23] H. W. Blanch, Proc. Int. Workshop on Biocatalysis in Nonconventional Media, Athens, 19-30 Oct., 1992

[24] M. Masoom, A. Mateen and A. Townshend, J. Chem. Soc. Pak. 11 (1989) 81.

[25] J.W. Shield, H.D. Ferguson, A.S. Bommarius and T.A. Hatton, Ind. Eng. Chem. Fundam. 25 (1986) 603.

[26] K. Markinek, I.V. Berezia, Y.L. Khmelnitski, N.L. Klyachko and A.V. Levashov, Coll. Czech. Chem. Commun. 52 (1987) 2589.

[27] H.F. Eicke, Top. Curr. Chem. 87 (1980) 85.
[28] D. Balasubramanian, J. Indian Chem. Soc. 58 (1981) 633.

[29] J.T.G. Overbeek, Proc. Kon. Ned. Akad. Wetenschap. B 89 (1986) 61.

[30] P.L. Luisi, M. Giomini, M.P. Pileni and B.H. Robinson, Biochim. Biophys. Acta 947 (1988) 209.

[31] P.L. Luisi and L.J. Magid, CRC Crit. Rev. Biochem. 20 (1986) 409.

[32] K. Martinek, A.V. Levashov, N.L. Klyachko, Y.L. Khmelnitski and I.V. Bcrezin, Eur. J. Biochem. 155 (1986) 453.

[33] Y.L. Khmelnitski, A.V. Levashov, N.L. Klyachko and K. Martinek, Russ. Chem. Rev. 53 (1984) 319.

[34] P.L. Luisi, Angew. Chem. Int. Ed. Engl. 24 (1985) 439.

[35] M. Waks, Proteins Structure Function Genetics 1 (1986) 4.

[36] P.L. Luisi and B. Steinmann-Hofmann, in: K. Mosbach (Ed.), Methods in Enzymology, Vol. 136 (Academic Press, New York, 1987) p. 188.

[37] Y.L. Khmelnitsky, A.V. Kabanov, N.L. Klyachko, A.V. Levashov and K. Martinek, in: M.P. Pileni (Ed.), Structure and Reactivity in Reverse Micelles Studies in Physical and Theoretical Chemistry, Vol. 65 (Elsevier, Amsterdam, 1989) p. 230.

[38] M.P. Pileni, in: M.P. Pileni (Ed.), Structure and Reactivity in Reverse Micelles Studies in Physical and Theoretical Chemistry, Vol. 65 (Elsevier, Amsterdam, 1989) p. 44.

[39] P.S. Chang, J.S. Rhee and J.J. Kim, Biotechnol. Bioeng. 38 (1991) 1159.

[40] D.M.S. Prazeres, F.A.P. Garcia and J.M.S. Cabral, Biotech. Bioeng. 41 (1993) 761.

[41] D.M.S. Prazeres, F.A.P. Garcia and J.M.S. Cabral, in: J. Tramper, M.H. Vermue, H.H. Beeftink and U. Stockar (Eds.), Biocatalysis in Non-conventional Media (Elsevier, Amsterdam, 1992) p. 713.

[42] D.M.S. Prazeres, F.A.P. Garcia and J.M.S. Cabral, Bioproc. Eng. 10 (1994) 21.

[43] M.L. Serralheiro, D.M.F. Prazeres and J.M.S. Cabral, Enz. Microb. Technol., in press.

[44] C.L. Chiang and S.W. Tsai, J. Chem. Tech. Biotechnol. 54 (1992) 27.

[45] P. Luthi and P.L. Luisi, J. Am. Chem. Soc. 106 (1984) 7285.

[46] C.L. Chiang and S.W. Tsai, J. Chem. Tech. Biotechnol. 54 (1992) 249.

[47] Y.L. Khmelnitsky, A.K. Gladilin, V.L. Roubailo, K. Martinek and A.V. Levashov, in: J. Tramper, M.H. Vermue, H.H. Beeftink and U. Stockar (Eds.), Fundamentals of Biocatalysis in Non-Conventional Media (Elsevier, Amsterdam, 1992).

[48] D.K. Eggers and H.W. Bloch, Bioprocess Eng. 3 (1988) 83.

[49] P.L. Luisi, P. Luthi, I. Tomka, J. Prenosil and A. Pande, Ann. N.Y. Acad. Sci. 434 (1988) 549.

[50] P. Luthi and T.A. Hatton, Bioseparation 2 (1991) 5.

[51] D.M.F. Prazeres, F. Lemos, F.A.P. Garcia and J.M.S. Cabral, Biotech. Bioeng. 42 (1993) 759.

[52] D.M.F. Prazeres, F. Lemos, F.A.P. Garcia and J.M.S. Cabral, Biotech. Bioeng. 42 (1993) 765.

[53] Y.L. Khmelnitsky, A.V. Levashov, N.L. Klyachko and K. Martinek, Enz. Microb. Technol. 10 (1988) 710.

[54] K. Magoulas and D. Tassios, Proc. Int. Workshop on Biocatalysis in Non-conventional Media, Athens, 19-30 Oct., 1992. 
[55] K. Nakamura, Y.M. Chi and Y. Yamada, Chem. Eng. Commun. 45 (1985) 207.

[56] T.W. Randolph, H.W. Blanch, J.M. Prausnitz and C.R. Wilke, Biotechnol. Lett. 7 (1985) 325.

[57] D.A. Hammond, M. Karel and A.M. Klibanov, Appl. Biochem. Biotechnol. 11 (1985) 393.

[58] Y.M. Chi and K. Nakamura, Agric. Eng. Chem. 52 (1988) 1541.

[59] A.M.M. van Eijs, J.P.L. de Jong, H.J. Doddema and D.R. Lindeboom, Proc. of the Int. Symp. on Supercritical Fluids, Nice, France, 1988, p. 933.

[60] D.A. Hammond, M. Karel and V.J. Krukonis, Appl. Biochem. Biotechnol. 11 (1985) 393.

[61] A. Marty, W. Chulalaksnanukal, J.S. Condoret, R.M. Willemot and G. Durand, Biotechnol. Lett. 12 (1990) 11.

[62] P. Pasta, G. Mazzola, G. Carrea and S. Riva, Biotechnol. Lett. 2 (1989) 643.

[63] T.W. Randolph, D.S. Clark, H.W. Blanch and J.N. Praunitz, Science (1988) 387.

[64] M. Taniguchi, M. Kamihira and T. Kobayashi, Agric. Biol. Chem. 51 (1987) 593.

[65] M. Perrut, in: C. Balny, R. Hayashi, K. Heremans and P. Masson (Eds.), High Pressure and Biotechnology, Colloque INSERM/John Libbey Eutotext, Vol. 224 (1992) p. 401.

[66] D. Combes and J.S. Condoret, Proc. Int. Workshop on Biocatalysis in Non-conventional Media, Athens, 19-30 Oct., 1992

[67] G.B. Lim, G.D. Holder and Y.T. Shah, in: K.P. Johnston and J.M.L. Penninger (Eds.), Supercritical Fluid Science and Technology (1989) p. 379.

[68] D. Combes, in: F.X. Malcata (Ed.), Engineering of/with Lipases (Kluwer Academic Publishers, Dordrecht, 1996) p. 613.

[69] A. Marty, D. Combes and J.S. Condoret, Biotechnol. Bioeng. 43 (1994) 497.

[70] D. Combes, in: F.X. Malcata (Ed.) Engineering of/with Lipases (Kluwer Academic Publishers, Dordrecht, 1996) p. 289.

[71] Anonymous, U.S. Patent $1,454,462$ (1923).

[72] Anonymous, U.S. Patent 1,454,463 (1923).

[73] K. Sennewald, K. Gehrmann and S. Schafer, U.S. Patent 3,579,309 (1971).

[74] A.L. Paiva and F.X. Malcata, in: F.X. Malcata (Ed.), Engi- neering of / with Lipases (Kluwer Academic Publishers, Dordrecht, 1996) p. 597.

[75] A.L. Paiva and F.X. Malcata, Biotechnol. Techn. 8 (1994) 629.

[76] T.H. Ching, Appl. Microb. Technol. 19 (1984) 1.

[77] J.A.M. De Bont and C.G. van Ginkel, Enz. Microb. Technol. 5 (1983) 55.

[78] F. Parvaresh, G. Vic, D. Thomas and M.D. Legoy, Ann. N.Y. Acad. Sci., in press.

[79] S. Pulvin, F. Parvaresh, D. Thomas and M.D. Legoy, Ann. N. Y. Acad. Sci. 542 (1988) 434.

[80] S. Pulvin, M.D. Legoy, R. Lortie, M. Pensa and D. Thomas, Biotechnol. Lett. 8 (1986) 783.

[81] E. Barzana, A. Klibanov and M. Karel, Appl. Biochem. Biotechnol. 15 (1987) 25.

[82] S. Pulvin, M.D. Legoy, R. Lortie, M. Pensa and D. Thomas, Biotechnol. Lett. 8 (1986) 783.

[83] S. Pulvin, F. Parvaresh, D. Thomas and M.D. Legoy, Ann. N.Y. Acad. Sci. 545 (1988) 434.

[84] F. Parvaresh, H. Robert, D. Thomas and M.D. Legoy, Biotechnol. Bioeng. 39 (1992) 467.

[85] H. Robert, S. Lamare, F. Parvaresh and M.D. Legoy, Prog. Biotechnol. 8 (1992) 23.

[86] S. Lamare and M.D. Legoy, Trends Biotechnol. 117 (1993) 413.

[87] S. Lamare and M.D. Legoy, Biotechnol. Bioeng. 45 (1995) 387.

[88] S. Lamare and M.D. Legoy, Biotechnol. Tech. 9 (1995) 127.

[89] E. Barzana, A. Klibanov and M. Karel, Appl. Biochem. Biotechnol. 1S (1987) 25.

[90] S. Lamare, in: F.X. Malcata (Ed.), Engineering of /with Lipases (Kluwer Academic Publishers, Dordrecht, 1996) p. 357.

[91] C. Laane, J. Tramper and M.D. Lilly (Eds.), Biocatalysis in Organic Media (Elsevier, Amsterdam, 1987).

[92] J. Tramper, M.H. Vermue, H.H. Beeftink and U. von Stockar (Eds.), Biocatalysis in Non-conventional Media (Elsevier, Amsterdam, 1992).

[93] A.L. Paiva and F. X. Malcata, Chem. Eng. Sci., 52 (1997) 449.

[94] A.L. Paiva, D. van Rossum and F.X. Malcata, Biocatalysis and Biotransformation, submitted. 